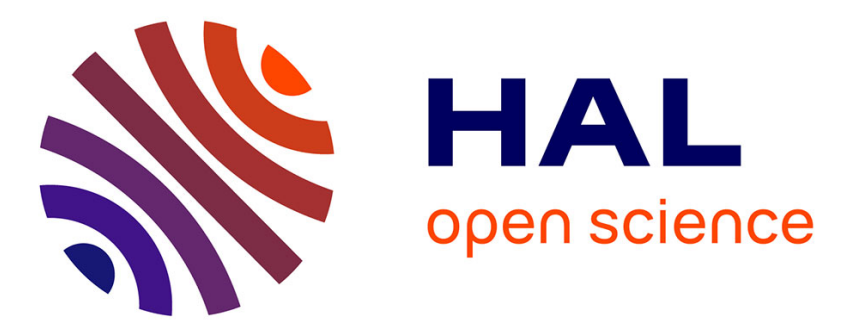

\title{
In vivo ankle joint kinematics from dynamic magnetic resonance imaging using a registration-based framework
} Karim Makki, Bhushan Borotikar, Marc Garetier, Sylvain Brochard, Douraied Ben Salem, François Rousseau

\section{- To cite this version:}

Karim Makki, Bhushan Borotikar, Marc Garetier, Sylvain Brochard, Douraied Ben Salem, et al.. In vivo ankle joint kinematics from dynamic magnetic resonance imaging using a registration-based framework. Journal of Biomechanics, 2019, 86, pp.193-203. 10.1016/j.jbiomech.2019.02.007 . hal02285921

\section{HAL Id: hal-02285921 \\ https://imt-atlantique.hal.science/hal-02285921}

Submitted on 13 Sep 2019

HAL is a multi-disciplinary open access archive for the deposit and dissemination of scientific research documents, whether they are published or not. The documents may come from teaching and research institutions in France or abroad, or from public or private research centers.
L'archive ouverte pluridisciplinaire HAL, est destinée au dépôt et à la diffusion de documents scientifiques de niveau recherche, publiés ou non, émanant des établissements d'enseignement et de recherche français ou étrangers, des laboratoires publics ou privés. 


\title{
In vivo Ankle Joint Kinematics from Dynamic Magnetic Resonance Imaging using a Registration-based Framework
}

\author{
Karim Makki ${ }^{a}$, Bhushan Borotikar ${ }^{b}$, Marc Garetier $^{c}$, Sylvain Brochard ${ }^{d}$, \\ Douraied Ben Salem ${ }^{e}$, François Rousseau ${ }^{a}$ \\ ${ }^{a}$ IMT Atlantique, LaTIM U1101 INSERM, UBL, Brest, France \\ ${ }^{b}$ University of Western Brittany, LaTIM U1101 INSERM, CHRU of Brest, Brest, France. \\ ${ }^{c}$ Service d'Imagerie Médicale, Hôpital d'Instruction des Armées Brest, LaTIM U1101 INSERM, France \\ ${ }^{d}$ Service de Médecine Physique et de Réadaptation, CHRU Brest, LaTIM U1101 INSERM, France \\ ${ }^{e}$ Service d'Imagerie Médicale, Hôpital de la Cavale Blanche, CHRU Brest, LaTIM U1101 INSERM, France
}

\begin{abstract}
In this paper, we propose a method for non-invasively measuring three-dimensional in vivo kinematics of the ankle joint from a dynamic MRI acquisition of a single range-of-motion cycle. The proposed approach relies on an intensity-based registration method to estimate motion from multi-plane dynamic MRI data. Our approach recovers not only the movement of the skeleton, but also the possibly non-rigid temporal deformation of the joint. First, the rigid motion of each ankle bone is estimated. Second, a four-dimensional (3D+time) high-resolution dynamic MRI sequence is estimated through the use of the log-euclidean framework for the computation of temporal dense deformation fields. This approach has been then applied and evaluated on in vivo dynamic MRI data acquired for a pilot study on six healthy pediatric cohort in order to establish in vivo normative joint biomechanics. Results demonstrate the robustness of the proposed pipeline and very promising high resolution visualization of the ankle joint.
\end{abstract}

Keywords : dynamic MRI, ankle, motion estimation, high-resolution reconstruction, intensity-based registration, locally rigid transformation

\section{Introduction}

To better understand the biomechanics of the pediatric ankle joint, it is crucial to establish in vivo normative joint biomechanics [7] before focusing on pathomechanics studies. This approach would likely contribute to a better long-term follow-up for childhood disabilities such as cerebral palsy.

Conventional MRI techniques have been used for accurate diagnosis of the ankle and foot disorders given the complexity of their anatomy [5]. Dynamic MRI was originally developed to study cardiovascular functions towards the end of the 1980s [18]. Since then, MRI acquisition and reconstruction techniques have improved by leaps and bounds with current sequences such as Fast-PC MRI, ultrafast MRI, and Fast Field Echo FFE. These techniques have been successfully employed to quantify the knee joint kinematics $[22,8]$, and to analyze in vivo 3D 
musculoskeletal dynamics [6, 25], non-invasively. Sequences such as cine-PC MRI [17, 26] or fast-PC MRI [23, 19] can provide in vivo joint velocity field measurements. However, these data require long acquisition times with a high number of repeated cycles. Sequences such as ultrafast MRI [9] allow faster scan speed by exploiting spatio-temporal redundancy and can acquire images during a single cycle. However, they require slow motion of the joint making the movement quasi-static. Thus, both these types of sequences are not appropriate to acquire dynamic in vivo data for children especially with musculoskeletal disorders. In this work, we use real-time FFE sequences [20] which are less sensitive to motion artifacts and which reduce the scanning time to 18 seconds to acquire the dorsi-plantarflexion cycle.

Furthermore, post-processing of acquired dynamic images remains a challenging task due to low resolution and motion artifacts. In [22], the displacement of specific points on each bone were quantified by integrating the velocity data using a three-dimensional Fourier tracking method [29]. Clarke et al. [9] determined in vivo muscle moment arms during ankle movement using a 3D mesh-based registration method. They estimated bone motions by registering the high-resolution joint geometry from MRI scans of the stationary joint to low-resolution geometries from ultrafast MRI scans of the slowly moving joint. However, in addition to the segmentation of the high-resolution static scan, it was needed to manually segment each low-resolution time-frame which was time-consuming and prone to segmentation bias.

In this study, the spatial information of the high-resolution static MRI data and the temporal information of the dynamic data were combined using a log-euclidean polyrigid framework $(L E P F)$. The purpose of this study was twofold : 1) to estimate bone motion, allowing to derive the 3D kinematics of the joint, 2) to compute temporal dense deformation fields of the joint, allowing to reconstruct a high-resolution dynamic MRI sequence from a low-resolution dynamic MRI sequence and one high-resolution MR image.

The use of the LEPF proposed by Arsigny et al. [4, 3, 2] has been investigated for registration of structures such as the hip and knee joint in mouse CT images [16], human hands from 2D hand radiographs [14], bones in lower-abdomen area [11], and also intra-subject mandible $3 \mathrm{D}$ datasets [21].

In this work, we adapted this framework to deal with 4-dimensional MRI data by proposing a fast and accurate algorithm to compute the dense deformation fields in regular grids. In fact, we propose to compute the exponential mapping of the LEPF using matrix eigendecomposition instead of the scaling and squaring method used in the literature $[2,11]$.

\section{Materials and methods}

\subsection{Subject recruitment}

Six typically developing children with a mean age of 12 years and with a mean weight of $35.8 \mathrm{~kg}$ participated in this study which was approved by the regional ethics committee. Children were selected with no contraindications to MRI and with no history of lower limb musculo-skeletal injury or surgery in the past six months. MRI data were acquired in a single visit after parents signed informed consent forms. 


\subsection{Data Acquisition}

MRI data have been acquired using a 3T MR scanner (Achieva dStream, Philips Medical Systems, Best, Netherlands). An MRI-compatible orthotic fixture was used to perform dynamic movements inside the scanner(Figure 1). Individual range-of-motion was checked by the cilnician outside the MRI scanner and stops were placed on wire guides for controlling the range-of-motion inside the scanner. Each dynamic scan lasted for 18 seconds and ankle joint was moved (actively or passively) through the range-of-motion for each subject within 18 seconds with a rotation speed of $4 \circ / \mathrm{s}$ to $5 \circ / \mathrm{s}$ (depending on the range). Both passive and active acquisitions were standardized for all subjects as detailed in Annexe A. Dynamic images were acquired in the oblique axis of the ankle motion. This was done by acquiring axial dynamic scans first and then obliquely orienting the scanning plane for images to be acquired in sagittal plane.

The scanning protocol included one high-resolution static 3D scan of the ankle joint with a resolution of $0.26 \times 0.26 \times 0.8 \mathrm{~mm}$ (T1-weighted gradient-echo, flip angle $10^{\circ}$, matrix $576 \times 576$, FOV $150 \mathrm{~mm} \times 150 \mathrm{~mm}$, TR/TE $7.81 / 2.75 \mathrm{~ms}$, mean acquisition duration : 424.32 s), and three low-resolution dynamic sequences per child : two passive sequences for repeatability measures and one active sequence, all acquired with knee angle kept at full extension (approximately between $0^{\circ}$ to $10^{\circ}$ ). Each sequence is composed of 15 time frames with a voxel size of $0.57 \times 0.57 \times 8 \mathrm{~mm}$ (flip angle $15^{\circ}$, matrix $352 \times 352$, FOV $200 \mathrm{~mm} \times 200 \mathrm{~mm}$, TR/TE 20.61/1.8 ms, acquisition duration : $18.98 \mathrm{~s}$ ).
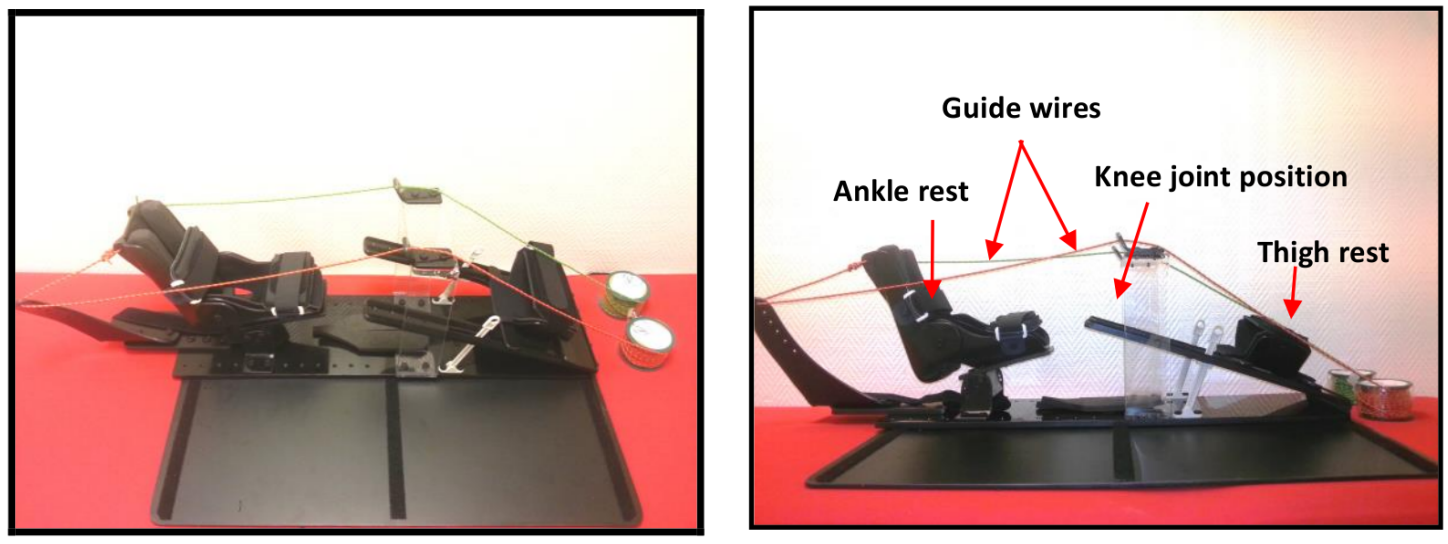

FIgURE 1 : Orthotic fixture specially designed to acquire MRI data on pediatric ankle joint. The entire fixture is made out of MRI-compatible material. Guide wires helped the technician to control and operate the passive plantar-dorsi flexion movements of the ankle joint while rest of the limb is fixed using straps at foot, tibia and mid thigh locations. Position of the ankle rest can be adjusted based on the limb length. Knee angle can be adjusted from full extension to $45^{\circ}$ flexion. Cushioned ankle rest supports the foot to be imaged.

\subsection{Data post-processing}

Temporal tracking of the ankle bones is a challenging task because of nonlinearly articulated joint motion. Image registration is an iterative process maximizing a similarity measure 


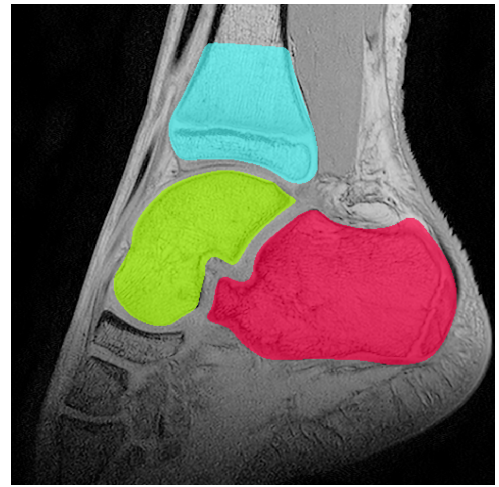

(a)

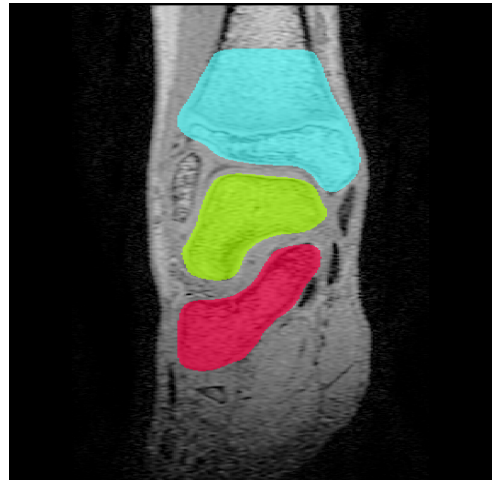

(b)

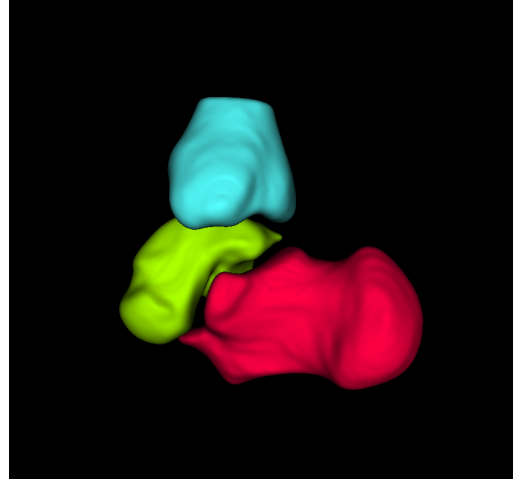

(c)

Figure 2 : Bones of interest : calcaneus (red), talus (green) and tibia (cyan). (a) : Mid-sagittal image from the high-resolution static scan; (b) : Mid-coronal image from the high-resolution static scan; (c) : Three dimensional rendering of segmented bones. 
step2 : The second step focused on the estimation of bone motion during the dynamic sequence. To do this, we proposed to take advantage of the temporal regularity of the data by propagating the bone masks in the dynamic sequence. For each bone, a rigid transform was estimated between successive images. i.e. local and rigid registrations were conducted from the reference image $D_{k^{*}}$ towards first and last images of the sequence. To go forward and backward in time, only one interpolation was needed to propagate segmentation from $D_{k^{*}}$ to each time frame $D_{k}$ by composing a set of successive rigid transforms in order to avoid the propagation of interpolation errors as much as possible. Temporal bone rotations were optimized based on their quaternion representations [15], making the segmentation propagation more robust.

The overall algorithm for bone motion estimation is described in Annexe B.

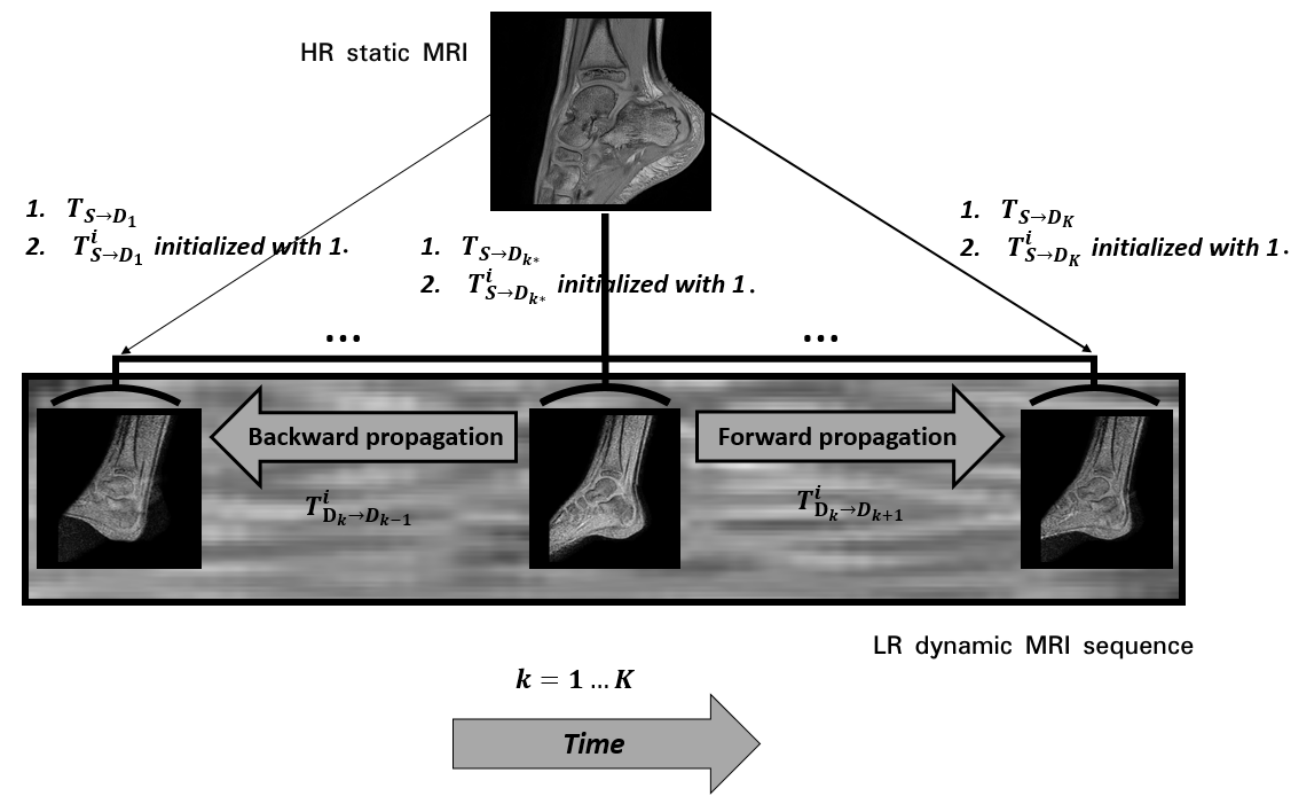

Figure 3: Proposed pipeline for ankle motion estimation : Bones of interest are segmented in the highresolution static image. This high-resolution MR image is globally (and rigidly) registered to each MR image of the dynamic sequence (1.), and the position of each bone is refined thanks to a local rigid registration using bone segmentations (2.). This allows to identify the dynamic MR image which is closest to the high resolution image and which will serve as a starting point for the motion estimation within the dynamic sequence. Last, local and rigid registrations are conducted from the reference towards the sequence first and last images.

\subsubsection{Computation of temporal dense deformation fields}

A dense deformation map of the joint from the static to each time frame was obtained by fusing rigid transforms corresponding to the bones of interest. For each bone, a non-negative weighting function was computed based on a distance to the mask [10]. Each weighting function reflects the local influence of the rigid transform of each bone. For each voxel $x$, the 
weighting functions were computed as follows :

$$
w_{D_{k}}^{i}(x)=\frac{1}{1+\operatorname{dist}^{2}\left(x, M_{D_{k}}^{i}\right)}
$$

where : $\operatorname{dist}\left(x, M_{D_{k}}^{i}\right)$ is the Euclidean distance between the point $x$ and the mask of the bone $i$ propagated onto the dynamic image $D_{k}$.

Given a point $x$ in the high-resolution static image, the target location of this point in the dynamic image $D_{k}$ can be computed with the following equation [4] :

$$
\mathcal{T}_{S \rightarrow D_{k}}(x)=\exp \left(\sum_{i=1}^{N} \tilde{w}_{D_{k}}^{i}(x) \log \left(T_{S \rightarrow D_{k}}^{i}\right)\right) \cdot x
$$

where $\mathcal{T}_{S \rightarrow D_{k}}$ is the dense invertible deformation field from the static image $S$ to the dynamic image $D_{k}, \tilde{w}_{D_{k}}^{i}$ is a normalized weighting function (i.e. $\sum_{i=1}^{N} \tilde{w}_{D_{k}}^{i}(x)=1, \forall x \in D_{k}$ ).

\subsubsection{Fast computation of dense deformation fields}

In this section, we describe an efficient algorithm to compute deformation fields in dense regular grid based on the algebraic properties of the Log-Euclidean polyrigid framework. In $[3,2]$, the matrix exponential was recursively approximated with a certain level of accuracy using the scaling and squaring method by taking into account that the matrix exponential is much simpler to compute for matrices close to zero via the Padé approximant. In this work, we computed the exponential mapping of equation 2 exactly using eigen decomposition. Assuming the log-euclidean mean of linear transformations $L(x)=\sum_{i=1}^{N} \tilde{w}_{D_{k}}^{i}(x) \log \left(T_{S \rightarrow D_{k}}^{i}\right)$ to be diagonalizable (i.e. $L(x)$ can be expressed in homogenous coordinates as : $L(x)=$ P.D. $P^{-1}(x)$, where the columns of $P$ contains the eigenvectors $\left\{v_{k}(x)\right\}_{k \in\{0 \ldots 4\}}$ corresponding to the complex eigenvalues of $\left.L(x),\left\{\lambda_{1}(x), \lambda_{2}(x), \lambda_{3}(x), 1\right\}\right)$, we broadcasted the exponentiation of transformation eigenvalues over all grid points using the following Equation :

$$
e^{L(x)}=P e^{D} P^{-1}(x)=P\left(\begin{array}{cccc}
e^{\lambda_{1}} & 0 & 0 & 0 \\
0 & e^{\lambda_{2}} & 0 & 0 \\
0 & 0 & e^{\lambda_{3}} & 0 \\
0 & 0 & 0 & e
\end{array}\right) P^{-1}(x)
$$

\subsection{Determination of ankle joint biomechanics}

\subsubsection{Definition of anatomically based coordinate systems}

Anatomical coordinate systems $\left\{R_{i}\right\}_{i=1, \ldots, N}$ were defined on each bone in the highresolution image $S$ following the same protocols used in [24] as illustrated in Figure 4, 
and then mapped into the neutral position $D_{n}$ using the estimated local-rigid transforms $\left\{T_{S \rightarrow D_{n}}^{i}\right\}_{i=1, \ldots, N}$. The neutral dorsi-plantarflexion position was defined at an ankle angle of $90^{\circ}$ as recommended by the ISB standards committee [28]. However, the above defined neutral position cannot be always achieved during dynamic scanning. Therefore, the image in which the foot position was closest to a dorsi-plantarflexion position of zero degrees was selected as the neutral position.

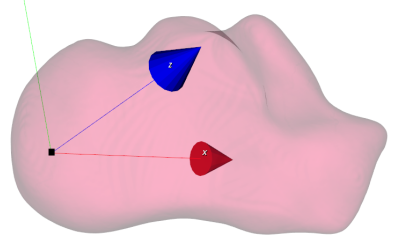

(a)

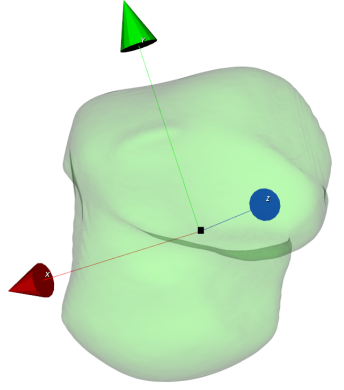

(b)

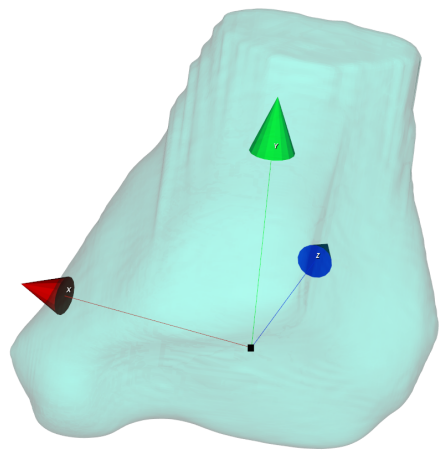

(c)

FIGURE 4 : Anatomical coordinate systems for one subject's calcaneus (a), talus (b), and tibia(c) as per ISB.

\subsubsection{Quantification of ankle joint kinematics}

In this study, the kinematics were defined through the 3D registration of the entire bone volume. More precisely, rotations and translations of each bone $i$ were determined from estimated rigid transforms $\left\{T_{D_{k} \rightarrow D_{k+1}}^{i}\right\}_{i=1, \ldots, N}$. Ranges of motion were estimated using low-resolution image data on multiple dynamic images (15 ankle positions). Kinematics of talocrural joint, subtalar joint, and calcaneal-tibial complex were then derived from $\left\{T_{D_{k} \rightarrow D_{k+1}}^{i}\right\}_{i=1, \ldots, N}$.

Given the image coordinate system $R_{I}=(O, \vec{x}, \vec{y}, \vec{z})$, we defined the transformation, $M_{R_{I} \rightarrow R_{j}}$, that mapped $R_{I}$ to an anatomical bone-based coordinate system $R_{j}=\left(O^{j}, \overrightarrow{x^{j}}, \overrightarrow{y^{j}}, \overrightarrow{z^{j}}\right)$. This transformation changed a representation from the $R_{j}$ system to the $R_{I}$ system.

Individual bone transformation matrices expressed in $R_{I}$ were then converted into the transformation matrices expressed in the new anatomical coordinate systems $R_{j}$ as follows :

$T_{j}^{i}(k)=\left(M_{R_{I} \rightarrow R_{j}} \times T_{D_{k} \rightarrow D_{k+1}}^{i} \times M_{R_{I} \rightarrow R_{j}}^{-1}\right)_{k=1, \ldots, K-1 ; i=1, \ldots, N ; \quad j=1, \ldots, N}$ where :

- $T_{j}^{i}(k): 4 \times 4$ rigid transformation matrix of the $i^{t h}$ bone relative to the $j^{t h}$ local bone coordinate system at time $k$.

- $M_{R_{I} \rightarrow R_{j}}$ : the change of basis matrix.

\subsection{Method evaluation}

To evaluate the proposed registration approach for bone motion estimation, the propagated mask of each bone through the dynamic sequence was compared with a manually delineated bone on each time frame. The accuracy of the estimations of the successive rigid 
transforms was assessed by computing the DICE coefficient Dice $\left(B_{k}^{i}, B_{m_{k}}^{i}\right)$ and the RMS error $R M S E\left(B_{k}^{i}, B_{m_{k}}^{i}\right)$ where $B_{k}^{i}$ was the propagated mask of the bone $i$ on dynamic image $D_{k}$ and $B_{m_{k}}^{i}$ was a manually delineated mask of the same bone on the dynamic image $D_{k}$. A DICE value close to one indicates that the bone masks have been well propagated through the entire dynamic sequence.

The RMS error was computed as follows :

$\operatorname{RMSE}\left(B_{k}^{i}, B_{m_{k}}^{i}\right)=\sqrt{1 / n_{c} \cdot \sum_{x_{c}=1}^{n_{c}} \operatorname{dist}^{2}\left(x_{c}, \zeta_{B_{k}^{i}}\right)}$ where $n_{c}$ is the total number of voxels of ground-truth contours (i.e. contours of $\left.B_{m_{k}}^{i}\right)$ and $\operatorname{dist}\left(x_{c}, \zeta_{B_{k}^{i}}\right)$ is the Euclidean distance between $x_{c}$ and the contour of $B_{k}^{i}$.

\section{Results}

Results on bone motion estimation were reported in Figures 8-9 for the six subjects of the pilot study. In most of the cases, the DICE coefficient was greater than 0.8 and the RMSE was smaller than 0.6 indicating accurate propagations of bone masks over the dynamic sequence, for both passive and active motions. From the set of rigid transforms of each bone over the dynamic sequence, it was then possible to compute temporal dense deformation fields to synthesize a high-resolution dynamic MRI sequence. Using the fast computation technique described in Section 2.3.3, a dense deformation field was computed in $3 \min$ on an Intel ${ }^{\circledR}$ Xeon ${ }^{\circledR}$ Processor E3-1271 v3 $3.60 \mathrm{GHz}$ on a $576 \times 576 \times 90$ regular grid and in $15 \mathrm{~min}$ on a very high dimensional space $(576 \times 576 \times 202 \simeq 67$ million deformation vectors $)$ which required a high capacity RAM because computations were performed in the complex domain for the purpose of enhancing the accuracy. Figure 10 illustrates, for one subject (A6), such high-resolution reconstructed data.

Three-dimensional kinematics of the joint under both active and passive ankle dorsiplantarflexion movement using the current 3D dynamic MRI method were presented in Figures 5 to 7 . Kinematics of all ankles (expect A5) were derived from the estimated temporal rigid transforms of each bone of interest, converted into the matrices of the calcaneus relative to the tibia, the talus relative to the tibia, and the calcaneus relative to the talus. The talus mistracking for the ankle A5 was caused by the considerable missing portion from the bone in intermediate time frames due to the inappropriate initial placement of the flex coils.

The mean rotation about the Inferior-Superior and about the Posterior-Anterior axes (averaged across time for all subjects) was close to 0 degrees for both active and passive motion. The mean range of passive rotation about the Medial- Lateral axis for talocrural an calcanealtibial joints was between -11 degrees of maximal dorsiflexion and +32 degrees of maximum plantarflexion with full extended knee. This demonstrates excellent correspondance with the mean range of passive rotation reported in [1] and validated for 245 healthy children between 7 and 14 years old. 

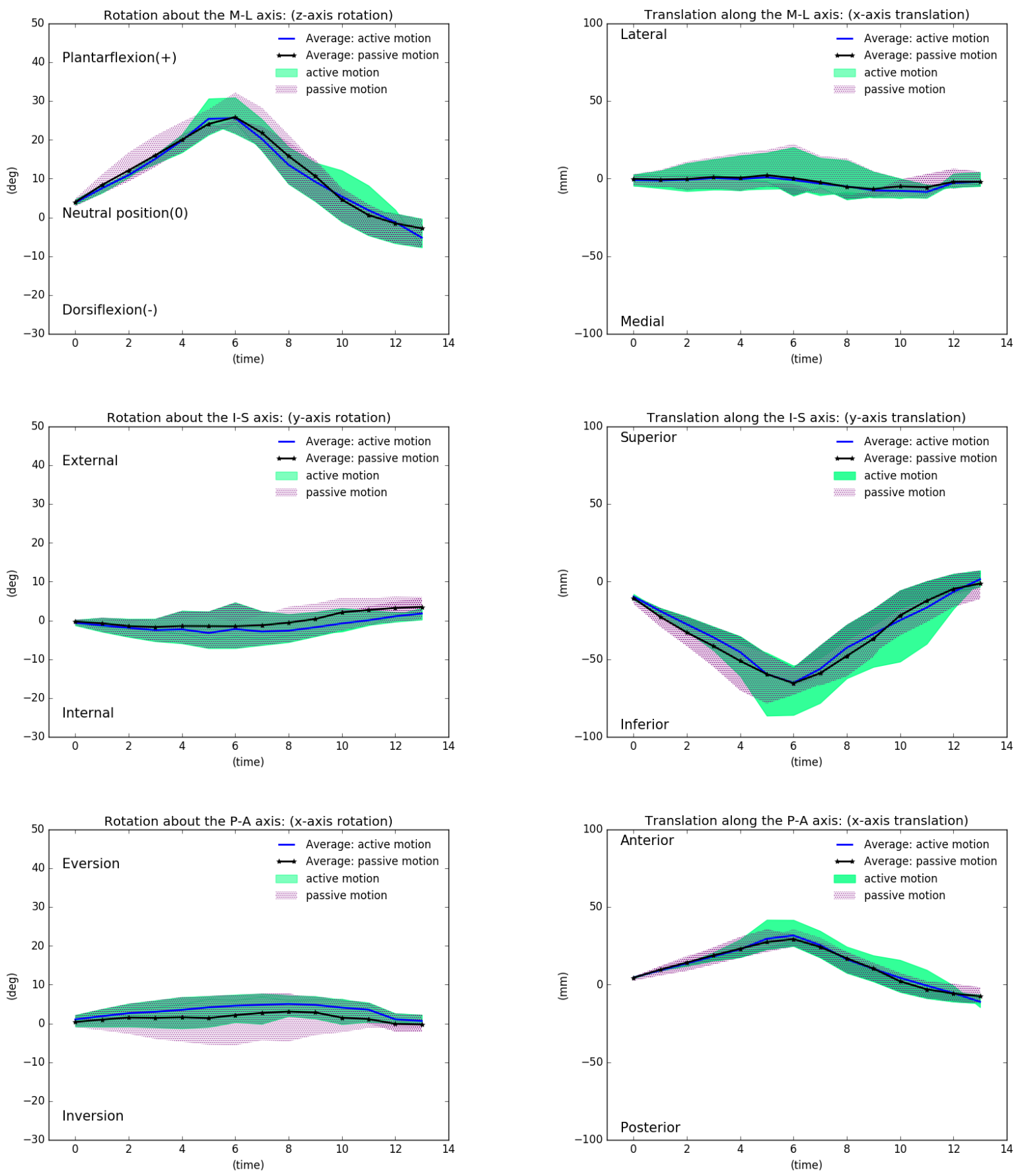

FIGURE $5: 3 \mathrm{D}$ normative kinematics of the calcaneal-tibial complex using the proposed image processing method. The kinematics of the calcaneus are represented relative to the tibial coordinate system defined in neutral position. Average rotation and translation were computed for both passive and active motion for the studied ankles. Standard deviation above and below the average line are shown (hatched shaded area for passive motion and green shaded area for active motion). 

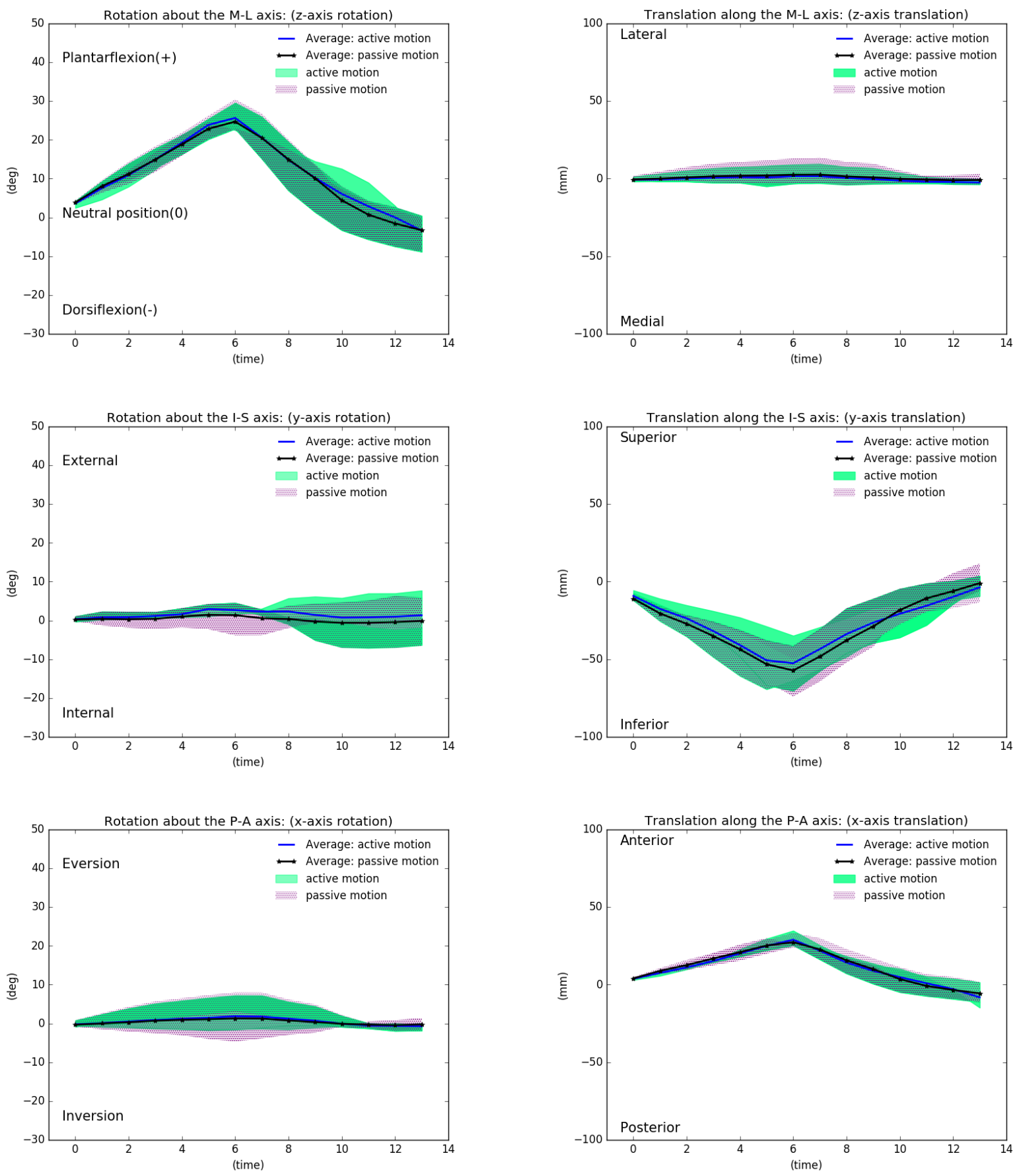

FIGURE $6: 3$ D normative kinematics of the talocrural (talar-tibial) joint using the proposed image processing method. The kinematics of the talus are represented relative to the tibial coordinate system in neutral position. Average rotation and translation were computed for both passive and active motion for the studied ankles. Standard deviation above and below the average line are shown (hatched shaded area for passive motion and green shaded area for active motion). 

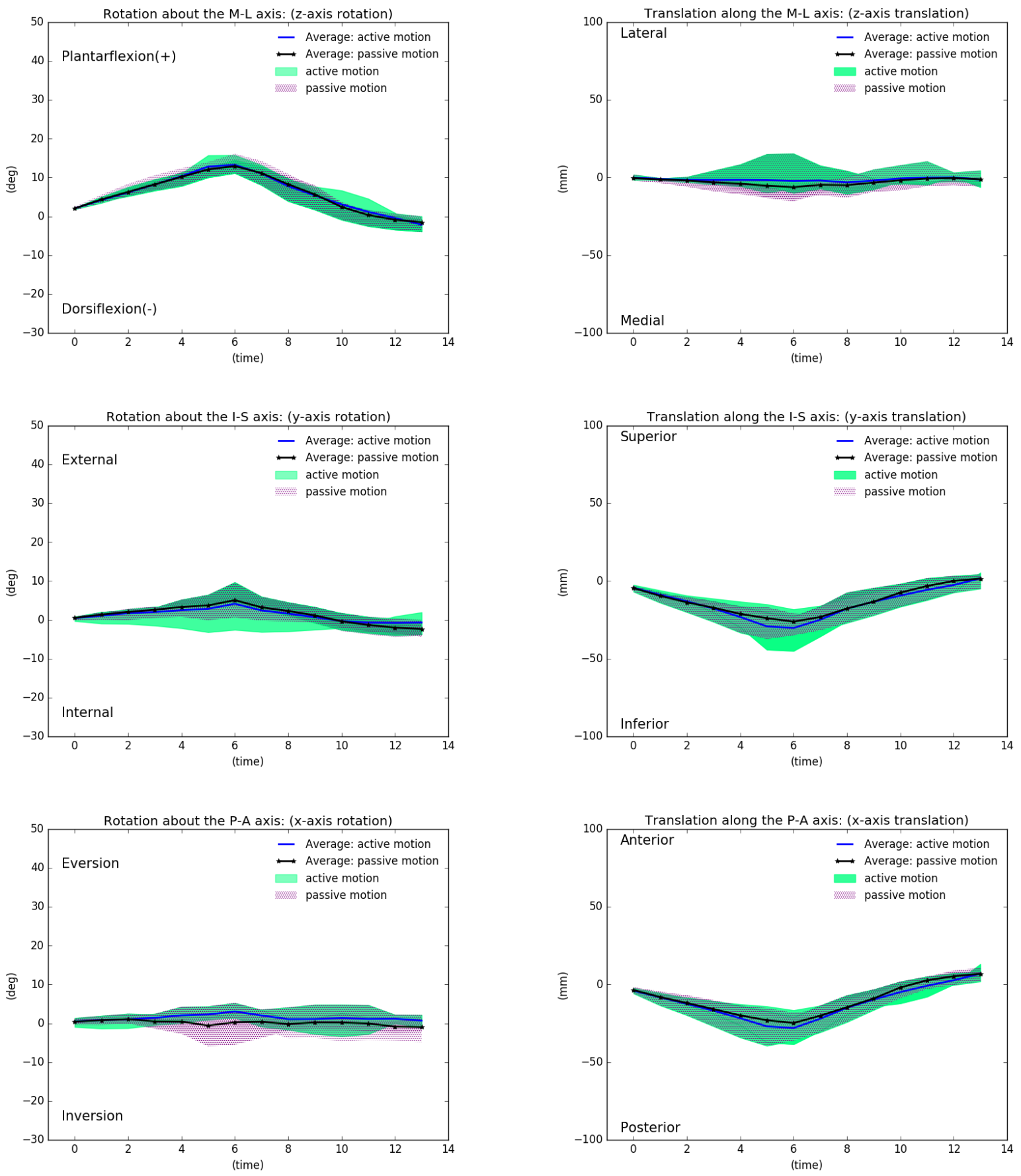

FIGURE 7 : 3D normative kinematics of the subtalar joint using the proposed image processing method. The kinematics of the calcaneus are represented relative to the talar coordinate system in neutral position. Average rotation and translation were computed for both passive and active motion for the studied ankles. Standard deviation above and below the average line are shown (hatched shaded area for passive motion and green shaded area for active motion). 

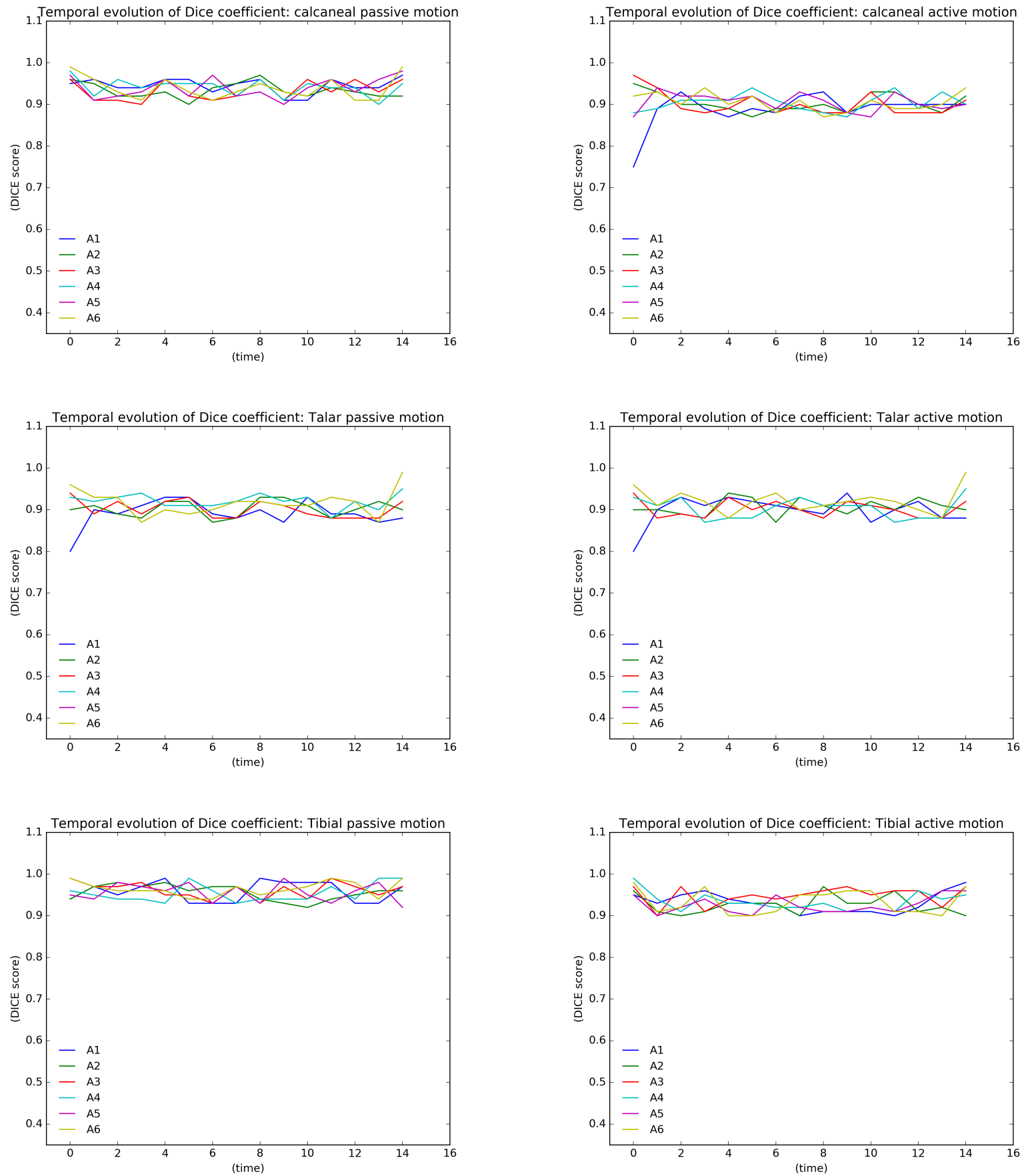

FigURE 8 : 3D DICE coefficients between manual and automatic bone segmentation maps for the six subjects of the pilot study taking account of the whole set of bone trajectories. Results for passive (left column) and active motion (right column) are separated. A dice value of 1 indicates perfect geometric alignment between automatic and ground truth segmentations. 

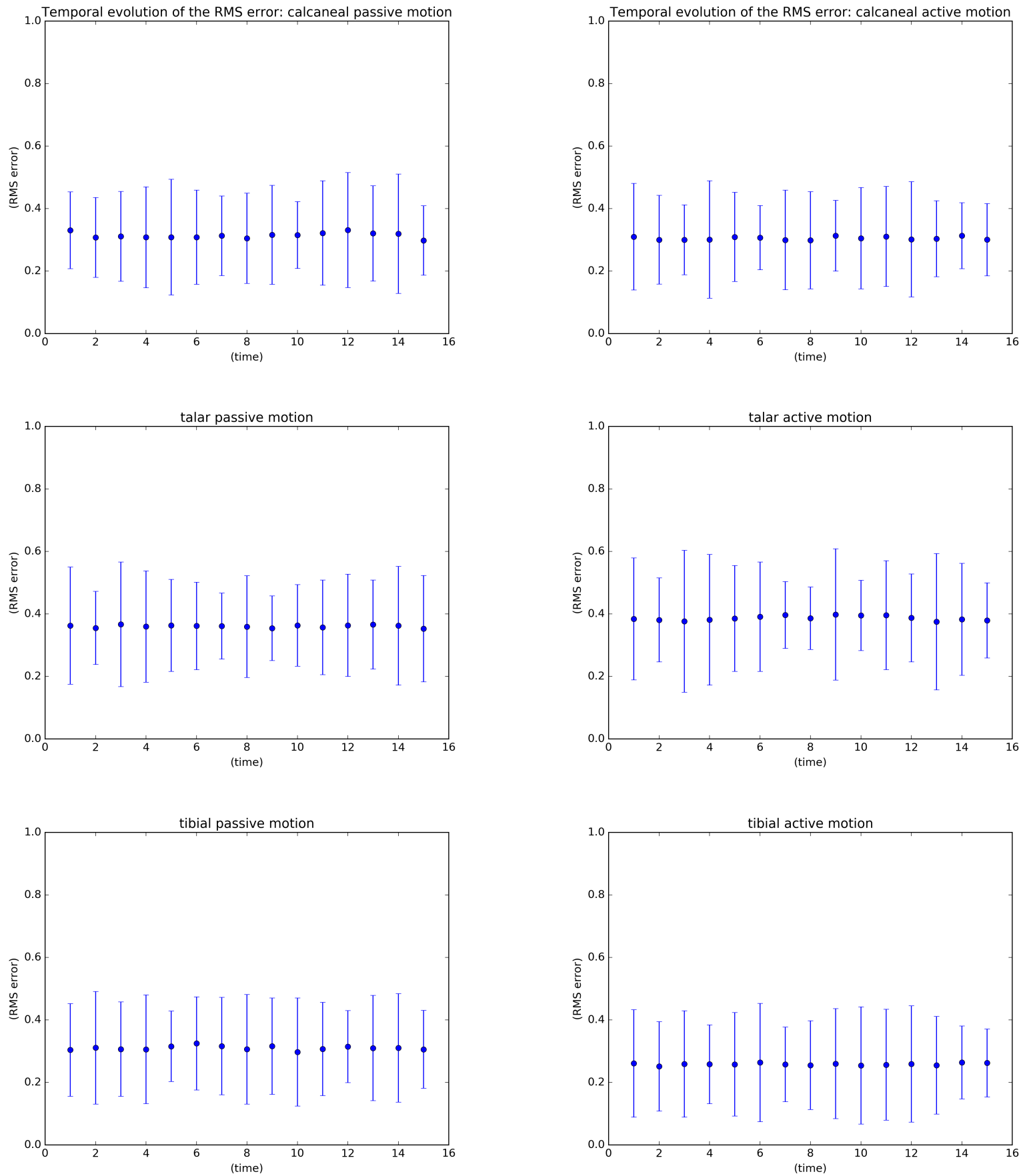

FiguRE 9 : Temporal evolution of the root-mean-square error (RMSE in voxels) between manual and automatic bone segmentations for subjects of the pilot study. Errors are represented using error bars. The average is shown with a dotted line with one standard deviation above and below this line. Results for passive (left column) and active motion (right column) are separated. 


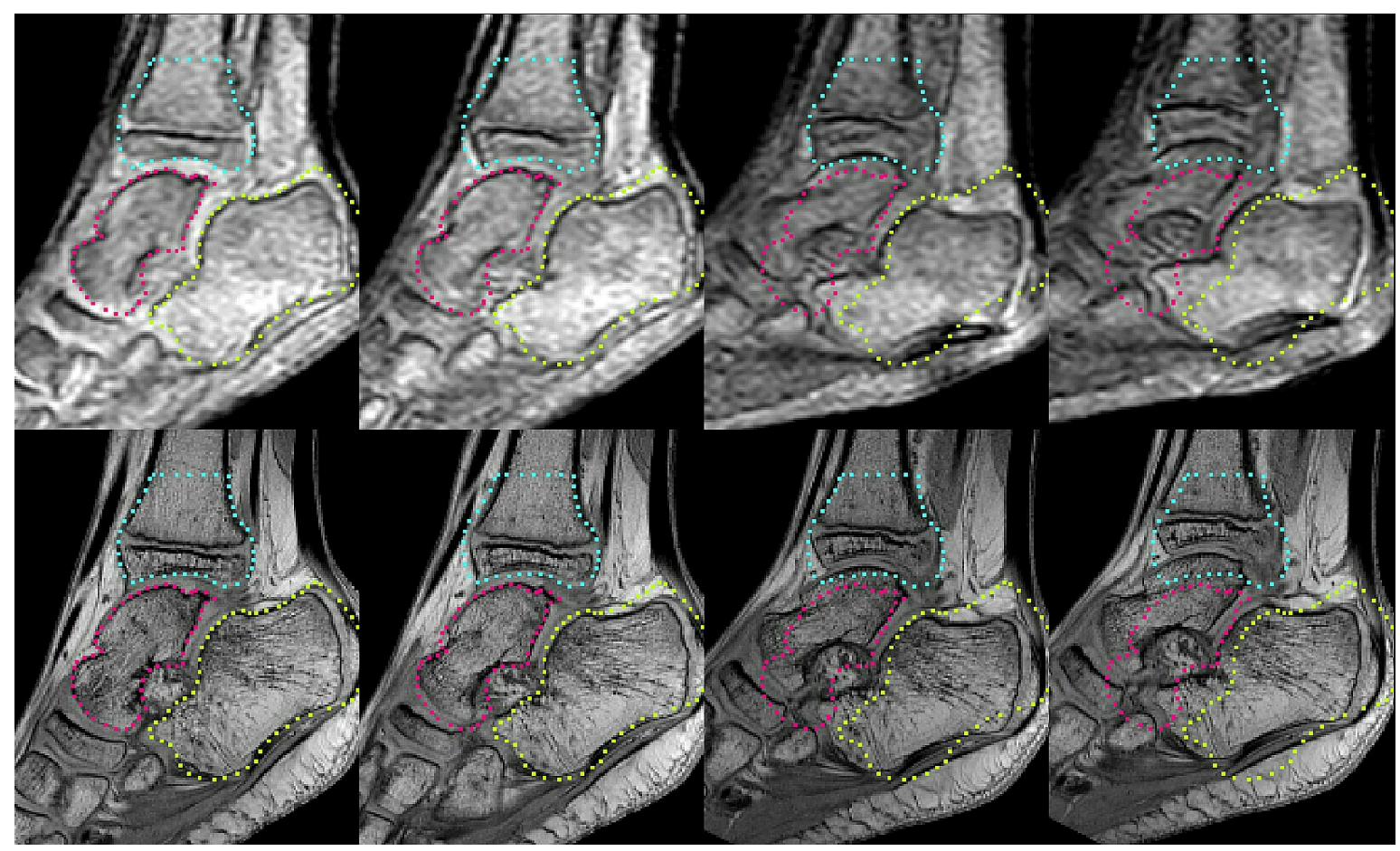

FigURE 10 : High resolution temporal reconstruction for one subject (subject 6 : passive motion). First row : original dynamic images, second row : corresponding high-resolution reconstructed images, downsampled to resemble to the original low-resolution images in order to validate the reconstruction accuracy. Each column corresponds to one time frame. Contours of the first time frame show the reconstruction accuracy (column 1) and the joint motion (columns 2 to 4 ).

\section{Discussion}

In this study, we have presented a method to determine full 3D-kinematics of the ankle joint from dynamic anatomical MRI data. An intensity-based registration pipeline has been proposed to estimate bone motion through the dynamic sequence. The robustness of this approach for both passive and active motion has been evaluated on in vivo data acquired for a pilot control cohort study. Although based on a successive estimation of transformations, the proposed approach demonstrates advantages in efficiency, reliability and robustness for all subjects.

The proposed algorithm is robust enough to image quality as well as motion artefacts in a way that it can determine kinematics even for unconstrained motion. This was evaluated by changing the delta-time $\Delta k$ separating each couple of source/target images during the successive rigid registrations and the algorithm is still robust even for $\Delta k=5$.

Normative passive kinematics show that the subjects maintained a consistent movement trajectory. Three-dimensional kinematics of the joint under passive ankle movement using the proposed method (Figures 5 to 7 ) were similar to ankle joint kinematics reported in other studies using velocity data [24]. Normative active kinematics show that the subjects 
were able to perform voluntary plantar-dorsiflexion between the extreme positions with the same temporal regularity as for passive motion. The comparison between passive and active kinematics shows closest temporal averages despite some temporal fluctuation of the standard deviation across subjects.

Although the fact that both our method and other motion methods tend toward the same goal which is bone motion tracking, there are some technical differences making it difficult to perform an objective comparison based on a common error parameter.

In [9], the authors were more interested in the Achilles tendon moment arms and the accuracy and precision of bone motion tracking were not reported. Clarke et al. [9] have discussed some limitations in their methods which can be particularly useful in adults. They have explicitly mentioned that their protocols require some modifications to be useful in the smaller joints of children. To satisfy the mesh density requirements for using the ICP algorithm, they have recommended the reduction of the slice gap for the dynamic scanning protocol while increasing the number of slices per time frame so that the slices span the joint width. However, assuming that they have used ultrafast MRI sequences (with 8 sagittal slices/time frame compared to 6 slices in our work, and taking around 2 min to acquire 10 to 20 time frames compared to only $18 \mathrm{sec}$ to acquire 15 time frames in our work), increasing the number of slices will increase the scanning time of dynamic data to more than 2 min which will be uncomfortable especially for the pathological subjects. The authors have also notified that the rotation speed for their study was slow (mean $1^{\circ} / \mathrm{s}$ compared to $4.2^{\circ} / \mathrm{s}$ in our work) in order to reduce the effects of motion artifacts, so the motion itself is reduced to almost static or quasi-static nature where no real functionality can be assessed. Our choice for FFE sequences for dynamic data acquisition was motivated by the fact that these techniques are less sensitive to motion artifacts [27] and more than 5 times faster than ultrafast MRI. In the work of Sheehan et al. [24], the displacements of three to five points on each bone were calculated through Fourier integration and then converted into three-dimensional orientation angles. As part of the tracking process, they identified a series of vertices, defining regions on each bone of interest in the first time frame (i.e. three to five vertices per bone). The distance between these vertices in the first time frame was considered the absolute distance under the assumption that it should remain fixed throughout the movement because bones are rigid. Thus, for each bone, the difference in distance between vertices in each time frame relative to the absolute distance was defined as the tracking error. A potential limitation of this study [24] was the out of plane accuracy of the bone motion because the accuracy of cine-PC MRI is independent of the shape of the bone being tracked [6]. Another clinical limitation is that subjects with musculoskeletal disease who cannot complete a large number of repeated motions cannot be studied with cine-PC MRI techniques. Although the vertices were well-defined from 3D high-resolution adult data in previous work of Sheehan et al. [24], it was hard to reproduce their exact locations from developing children data for comparison purposes because of the differences in bone size and topology between children and adults. From another point of view, it was not sufficient to consider the distance between only some vertices as system bias and thus we have considered all the bone segmentations (i.e. all the 
volumetric information) for method evaluation.

The proposed method remains robust as long as the field-of-view (FOV) is well adjusted (i.e. when the FOV covers either the full or nearly-full anatomy of the ankle bones throughout the entire joint trajectory).

We have also extended the log-euclidean framework to estimate temporal dense deformation fields from multiple rigid transforms. The polyrigid approach provides a way to combine high resolution spatial information with temporal dynamics of joints. The output of this process is a series of high-resolution anatomical images portraying the different phases of the movement cycle. In this context, we proposed to compute the exponential mapping of the LEPF in an efficient and elegant way using matrix diagonalization-based techniques rather than using techniques involving approximation theory. The preservations of the bone shapes after the polyrigid fusion were checked by computing the Jacobian maps associated to the estimated deformation fields (as shown in figure 11).

To conclude, our work is the first effort to track ankle joint motion and deformation from volumetric image data using intensity-based non-rigid image registration rather than using explicit mechanistic models. It therefore achieves its goals without having to perform time-consuming manual segmentations. In immediate future, the proposed approach will be applied to compare the ankle joint dynamics of children with spastic equinus with age-matched healthy children. Since spastic equinus is typically defined as the inability to dorsiflex the foot above plantigrade, with the hindfoot in neutral position and the knee in extended position [13], the foot was constrained to a specific path using the fixture in this work. However, this set-up is problem-specific and can be changed or removed while evaluating the pathomechanics of other joint(s). We will also explore this method to extract fine biomechanical parameters of tendon, and cartilage contact mechanics (e.g. temporal joint space width) of the tibio-talar joint which is the primary joint responsible for plantarflexion and dorsiflexion of the ankle. Furthermore, these techniques can be applied to other joints and related musculoskeletal disorders. This suggests that anatomical dynamic MRI and dedicated image processing techniques can open a new way to study in vivo human joints.

\section{Acknowledgments}

The research leading to these results has received funding from Région Bretagne, Chaire d'excellence INSERM-IMT Atlantique, Fondation de l'Avenir, Paris, France, and Fondation Motrice, Paris, France.

\section{References}

[1] Alanen, J.T., Levola, J.V., Helenius, H.Y., Kvist, M.H.. Ankle joint complex mobility of children 7 to 14 years old. Journal of Pediatric Orthopaedics $2001 ; 21(6)$ :731-737. 


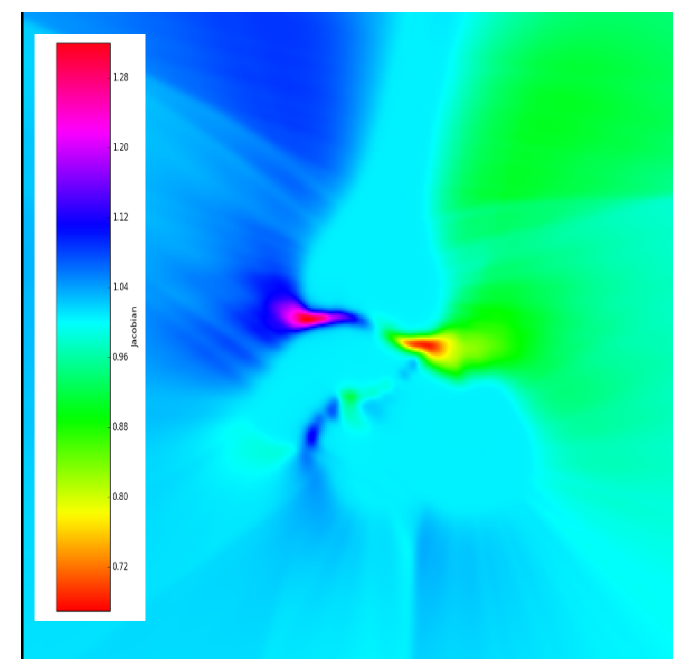

Figure 11 : Jacobian map of the joint deformation fields that maps $D_{1}$ to $D_{2}$ for subject 4 . The Jacobian determinant $J(x)$ at a voxel $x$ measures how the voxel volume changes after registration. It indicates a volume increase if $>1$, and a volume decrease if $<1$. The Jacobian determinant is equal to 1 inside bone segmentations (no voxel volume changes) as bones only perform linear rigid transformations (rotations and translations).

[2] Arsigny, V., Commowick, O., Ayache, N., Pennec, X.. A fast and log-euclidean polyaffine framework for locally linear registration. Journal of Mathematical Imaging and Vision $2009 ; 33(2): 222-238$.

[3] Arsigny, V., Commowick, O., Pennec, X., Ayache, N.. A log-euclidean polyaffine framework for locally rigid or affine registration. In : International Workshop on Biomedical Image Registration. Springer ; 2006. p. 120-127.

[4] Arsigny, V., Pennec, X., Ayache, N.. Polyrigid and polyaffine transformations : a novel geometrical tool to deal with non-rigid deformations-application to the registration of histological slices. Medical Image Analysis $2005 ; 9(6): 507-523$.

[5] Bae, W., Ruangchaijatuporn, T., Chung, C.. New techniques in MR imaging of the ankle and foot. Magnetic Resonance Imaging Clinics 2017 ;25(1):211-225.

[6] Behnam, A.J., Herzka, D.A., Sheehan, F.T.. Assessing the accuracy and precision of musculoskeletal motion tracking using cine-PC MRI on a $3.0 \mathrm{~T}$ platform. Journal of biomechanics $2011 ; 44(1): 193-197$.

[7] Borotikar, B., Lempereur, M., Lelievre, M., Burdin, V., Ben Salem, D., Brochard, S.. Dynamic MRI to quantify musculoskeletal motion : A systematic review of concurrent validity and reliability, and perspectives for evaluation of musculoskeletal disorders. PLoS One $2017 ; 12(12)$ :e0189587.

[8] Borotikar, B.S., Sipprell, W.H., Wible, E.E., Sheehan, F.T.. A methodology to accurately quantify patellofemoral cartilage contact kinematics by combining $3 \mathrm{D}$ image shape 
registration and cine-PC MRI velocity data. Journal of biomechanics $2012 ; 45(6)$ :11171122.

[9] Clarke, E., Martin, J., d'Entremont, A., Pandy, M., Wilson, D., Herbert, R.. A non-invasive, 3D, dynamic MRI method for measuring muscle moment arms in vivo : demonstration in the human ankle joint and achilles tendon. Medical Engineering \& Physics $2015 ; 37(1): 93-99$.

[10] Commowick, O., Arsigny, V., Costa, J., Ayache, N., Malandain, G.. An efficient locally affine framework for the registration of anatomical structures. In : Biomedical Imaging : Nano to Macro, 2006. 3rd IEEE International Symposium on. IEEE; 2006. p. $478-481$.

[11] Commowick, O., Arsigny, V., Isambert, A., Costa, J., Dhermain, F., Bidault, F., Bondiau, P.Y., Ayache, N., Malandain, G.. An efficient locally affine framework for the smooth registration of anatomical structures. Medical Image Analysis 2008 ;12(4) :427441.

[12] Jenkinson, M., Bannister, P., Brady, M., Smith, S.. Improved optimization for the robust and accurate linear registration and motion correction of brain images. Neuroimage $2002 ; 17(2): 825-841$.

[13] Kay, R.M., Rethlefsen, S.A., Ryan, J.A., Wren, T.A.. Outcome of gastrocnemius recession and tendo-achilles lengthening in ambulatory children with cerebral palsy. Journal of Pediatric Orthopaedics B $2004 ; 13(2): 92-98$.

[14] Martín-Fernández, M., Martín-Fernández, M., Alberola-López, C.. A log-euclidean polyaffine registration for articulated structures in medical images. In : MICCAI(2009). Springer ; 2009. p. 156-164.

[15] Mukundan, R.. Quaternions : From classical mechanics to computer graphics, and beyond. In : Proceedings of the 7th Asian Technology conference in Mathematics. 2002. p. (2002) 97-105.

[16] Papademetris, X., Dione, D., Dobrucki, L., Staib, L., Sinusas, A.. Articulated rigid registration for serial lower-limb mouse imaging. In : MICCAI(2005). Springer; 2005. p. 919-926.

[17] Pelc, N.J., Herfkens, R.J., Shimakawa, A., Enzmann, D.R., et al. Phase contrast cine magnetic resonance imaging. Magnetic resonance quarterly $1991 ; 7(4): 229-254$.

[18] Pettigrew, R.. Dynamic cardiac MR imaging. Techniques and applications. Radiologic Clinics of North America $1989 ; 27(6): 1183-1203$.

[19] Rebmann, A.J., Sheehan, F.T.. Precise 3D skeletal kinematics using fast phase contrast magnetic resonance imaging. Journal of Magnetic Resonance Imaging $2003 ; 17(2)$ :206213. 
[20] Schaeffter, T.. Projection reconstruction balanced fast field echo for interactive realtime cardiac imaging. Magn Reson Med $2001 ; 46$ :1238-1241.

[21] Seiler, C., Pennec, X., Reyes, M.. Capturing the multiscale anatomical shape variability with polyaffine transformation trees. Medical Image Analysis 2012 ;16(7) :13711384.

[22] Sheehan, F.T., Babushkina, A., Alter, K.E.. Kinematic determinants of anterior knee pain in cerebral palsy : a case-control study. Archives of physical medicine and rehabilitation $2012 ; 93(8): 1431-1440$.

[23] Sheehan, F.T., Drace, J.E.. Quantitative MR measures of three-dimensional patellar kinematics as a research and diagnostic tool. Medicine and science in sports and exercise $1999 ; 31(10): 1399-1405$.

[24] Sheehan, F.T., Seisler, A.R., Siegel, K.L.. In vivo talocrural and subtalar kinematics : a non-invasive 3D dynamic MRI study. Foot \& ankle international $2007 ; 28(3): 323-335$.

[25] Sheehan, F.T., Smith, R.M.. 3d musculoskeletal kinematics using dynamic mri. Handbook of human motion $2017 ;: 1-17$.

[26] Sheehan, F.T., Zajac, F.E., Drace, J.E.. Using cine phase contrast magnetic resonance imaging to non-invasively study in vivo knee dynamics. Journal of biomechanics $1997 ; 31(1): 21-26$.

[27] Stehning, C., Börnert, P., Nehrke, K., Eggers, H., Dössel, O.. Fast isotropic volumetric coronary MR angiography using free-breathing 3D radial balanced FFE acquisition. Magnetic Resonance in Medicine : An Official Journal of the International Society for Magnetic Resonance in Medicine 2004 ;52(1) :197-203.

[28] Wu, G., Siegler, S., Allard, P., Kirtley, C., Leardini, A., Rosenbaum, D., Whittle, M., D D'Lima, D., Cristofolini, L., Witte, H., et al. ISB recommendation on definitions of joint coordinate system of various joints for the reporting of human joint motion - part i : ankle, hip, and spine. Journal of biomechanics $2002 ; 35(4)$ :543-548.

[29] Zhu, Y., Pelc, N.J.. Three-dimensional motion tracking with volumetric phase contrast MR velocity imaging. Journal of Magnetic Resonance Imaging : An Official Journal of the International Society for Magnetic Resonance in Medicine $1999 ; 9(1): 111-118$.

\section{Annexe A. Supplementary Material}

An MRI safe orthotic fixture has been specifically designed in such way that it can be used for performing either passive plantar-dorsiflexion or voluntary active ankle joint movement (Figure 1). For passive acquisition, after placing the ankle joint in the fixture, each child was asked to relax the lower-limb musculature and then the fixture was cyclically moved by a technician at a rotation speed of $4^{\circ} / \mathrm{s}$ to $5^{\circ} / \mathrm{s}$. The technician was given a set of headphones 


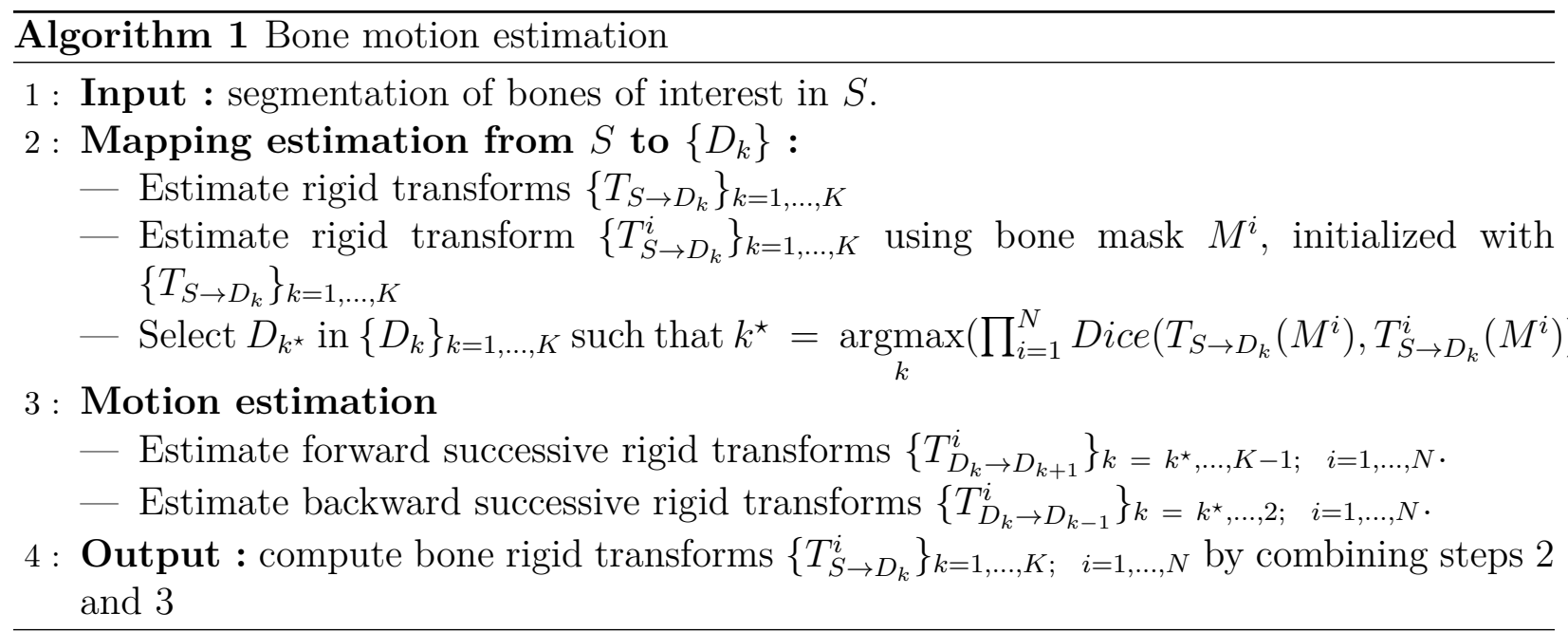

through which he/she could hear a metronome. Fixture was then moved in a consistent passive plantar-dorsiflexion by the technician at the beat of the metronome and using the guide wires attached to the fixture. For active movement, no technician was present and children were asked to perform voluntary plantar-dorsiflexion between the extreme positions on the beat of the metronome. Meaning, the active motion was completely voluntary with all the muscles dynamically taking part in the production of the plantar-dorsiflexion movement.

\section{Annexe B. Algorithm}

The overall algorithm for bone motion estimation is described in Algorithm 1 and in Figure 3, using the following notations :

$S$ : high-resolution static image, $\left\{D_{k}\right\}_{k=1, \ldots, K}$ : set of low-resolution dynamic images, $\left\{M^{i}\right\}_{i=1, \ldots, N}$ : set of mask of bones of interest, $T_{A \rightarrow B}$ : rigid transform from image $A$ to image $B, T_{A \rightarrow B}^{i}$ : rigid transform from image $A$ to image $B$ for the bone $i$, Dice(A,B) is the DICE coefficient which measures the overlap between segmented regions $\left(\operatorname{Dice}(A, B)=\frac{2|A \cap B|}{|A|+|B|}\right)$. Rigid registrations were performed using FSL-flirt [12] with correlation ratio as similarity measure. A voxel-wise weighting based on the segmentation masks was used when estimating bone-dependent motion.

Code is available online at https://github.com/rousseau/dynMRI. 